\title{
Visual Supernova Searching with the 40 inch Telescope at Siding Spring Observatory
}

\author{
Rev. Robert Evans \\ Uniting Church in Australia, 63 Cassilis St, Coonabarabran, \\ NSW 2357, Australia. \\ Received 1996 July 23, accepted 1997 April 18
}

\begin{abstract}
Preliminary results are presented arising from the use of the 40 inch telescope at Siding Spring Observatory for visual supernova hunting over a period of about 18 months. The use of the telescope in this way is continuing. These results are compared with the performance of my $41 \mathrm{~cm}$ backyard telescope over the same 18-month period, and with recently announced results from the Perth Observatory's Automated Supernova Search using their $61 \mathrm{~cm}$ telescope over a three-year period.
\end{abstract}

Keywords: supernovae - stars: statistics

\section{Introduction}

For some years until the beginning of 1986, a visual search for bright supernovae was conducted by the author from various locations with a second-grade quality $25 \mathrm{~cm}$ telescope. Twelve supernovae were eventually found with this telescope, and several others were observed that had been found first by others. Since that time, the search has been continued using a $41 \mathrm{~cm}$ telescope. Sixteen supernovae have been discovered with the $41 \mathrm{~cm}$ telescope, and about the same number of other supernovae have been observed with it. The ratio of those discovered to those merely observed has changed because the competition for discoveries has become greater in recent years.

Since 1986, part of the competition has been in the form of professional computerised automated searches using CCDs which have been conducted with larger telescopes, in particular, a $76 \mathrm{~cm}$ telescope at Berkeley, and now a $61 \mathrm{~cm}$ telescope at Perth. Several small-scale operations at other locations have also come into existence recently. At the Christchurch meeting of the Astronomical Society of Australia in 1993 I presented a paper which tried to compare the results of the Berkeley automated search from 1986 to mid-1991 (Muller et al. 1992) with my $41 \mathrm{~cm}$ search over the same period (Evans 1994). A total of twenty supernovae were seen at Berkeley compared to seventeen seen from my home. A difference of only three supernovae in the two searches over a period of nearly six years raised the possibility that, if a visual search had been conducted with a telescope like the one used at Berkeley, and assuming that the same amount of telescope time was available which the Berkeley team enjoyed, perhaps similar results might have been obtained by the visual search, or it might have been even more fruitful.

In order to test these ideas, a funding proposal through the Australian Research Council was launched in 1994 for a dedicated $1 \mathrm{~m}$ telescope in Australia, but this was not successful. This was followed by applications for observing time on the 40 inch telescope at Siding Spring. The Director of Mount Stromlo and Siding Spring Observatories, Professor Jeremy Mould, and the Time Allocation Committee have supported this plan even though there is steady professional competition for use of the telescope. This support was particularly noteworthy because the search was visual and did not use the regular $\mathrm{CCD}$ equipment. It was also totally staffed by amateur astronomers.

\section{Characteristics of this Search}

The aim has been to produce statistics of visual supernova searching with a $1 \mathrm{~m}$ class telescope that could be compared with what can be achieved using a smaller telescope, and also with the performance of a professional automatic search.

The method is simply to observe as many different galaxies as possible as regularly as possible. At least two people have been needed all the time on the 40 inch telescope: one to operate the telescope and one to observe. Optically we found that this telescope is an excellent instrument for this work. The most difficult and time-consuming part of the operation was locating the next object. This problem arises from the particular control system which is presently used with this telescope, related to the fact that it is thirty years old. We took our share of bright moonlight and bad weather which naturally reduced the potential of the large telescope visual search. This highlights the desirability of using a dedicated 
telescope which would allow darker nights and better conditions to be chosen.

\section{Statistics - so far}

Basic results are set out in Tables 1, 2 and 3. The search commenced with four nights at the end of 1994. During 1995 a total of 52 nights were allocated. So far, during 1996 (up

Table 1. Observing with the 40 inch telescope listed by calendar year

\begin{tabular}{lccc}
\hline Year & $\begin{array}{c}\text { Usable obs. } \\
\text { hours }\end{array}$ & $\begin{array}{c}\text { Galaxies } \\
\text { observed }\end{array}$ & $\begin{array}{c}\text { Supernovae } \\
\text { seen }\end{array}$ \\
\hline 1994 & 26 & 571 & 1 \\
1995 & 207 & 5223 & 5 \\
1996 & 140 & 4015 & 3 \\
& & & 9 \\
Total & 373 & 9809 & 9 \\
\hline
\end{tabular}

Table 2. Comparison of the three supernovae searches

\begin{tabular}{|c|c|c|c|c|}
\hline & & Perth & 40 inch & $41 \mathrm{~cm}$ \\
\hline \multirow[t]{5}{*}{ Mag. limit for SN } & & $16 \cdot 5-17 \cdot 0$ & & \\
\hline & Best conditions & & $17 \cdot 5+$ & $16 \cdot 5$ \\
\hline & Normal & $16 \cdot 5-17 \cdot 0$ & $15 \cdot 5-16 \cdot 0$ & \\
\hline & $\begin{array}{l}\text { Bright moon, } \\
\text { good seeing }\end{array}$ & $16 \cdot 0$ & & \\
\hline & $\begin{array}{c}\text { Bright moon, } \\
\text { bad seeing }\end{array}$ & $15 \cdot 0$ & & \\
\hline Period & & 3 years & $18 \mathrm{mths}$ & 18 mths \\
\hline Good quality observed & (hours) & $369+$ & 373 & 231 \\
\hline Number of galaxies observed & & 5530 & 9809 & 13,267 \\
\hline Observing rate & (galaxies/hr) & 15 & $26 \cdot 3$ & $57 \cdot 4$ \\
\hline Total SNe seen & & $5-6$ & 9 & 6 \\
\hline Discoveries & & $5-6$ & 2 & 2 \\
\hline Other $\mathrm{SNe}$ seen & & 0 & 7 & 4 \\
\hline \multirow{2}{*}{ Rate of seeing $\mathrm{SNe}$} & $(\mathrm{hr} / \mathrm{SN})$ & 61 & 41 & 38 \\
\hline & $($ galaxy/SN $)$ & 922 & 1022 & 2211 \\
\hline
\end{tabular}

Table 3. Details of observed supernovae

\begin{tabular}{|c|c|c|c|c|c|c|}
\hline Supernova & Details & $\begin{array}{c}\text { Perth } \\
\text { discovered }\end{array}$ & $\begin{array}{c}40 \text { inch } \\
\text { discovered }\end{array}$ & $\begin{array}{l}40 \text { inch } \\
\text { other seen }\end{array}$ & $\begin{array}{c}41 \mathrm{~cm} \\
\text { discovered }\end{array}$ & $\begin{array}{l}41 \mathrm{~cm} \\
\text { other seen }\end{array}$ \\
\hline SN 1993K & $\begin{array}{l}\text { NGC } 2223 \\
15 \mathrm{mag}\end{array}$ & $*$ & & & & \\
\hline SN 1994R & $\begin{array}{l}\text { IC } 2627 \\
15 \mathrm{mag}\end{array}$ & * & & & & \\
\hline $\begin{array}{l}\text { SN 1994ae } \\
\text { SN 1994ai }\end{array}$ & $\begin{array}{l}\text { NGC } 3370 \\
\text { NGC } 908 \\
16 \cdot 5\end{array}$ & * & & * & & * \\
\hline $\begin{array}{l}\text { SN 1995D } \\
\text { SN 1995G }\end{array}$ & $\begin{array}{l}\text { NGC } 2962 \\
\text { NGC } 1643 \\
\text { IAUC } 6138\end{array}$ & & * & $*$ & & $*$ \\
\hline SN $1995 \mathrm{H}$ & NGC 3526 & & & $*$ & & \\
\hline SN $1995 \mathrm{~V}$ & $\begin{array}{l}\text { NGC } 1087 \\
\text { IAUC } 6197\end{array}$ & & $*$ & & & * \\
\hline SN 1995W & $\begin{array}{l}\text { NGC } 7650 \\
16 \cdot 5 \mathrm{mag}\end{array}$ & * & & & & \\
\hline SN 1995ad & $\begin{array}{l}\text { NGC } 2139 \\
\text { IAUC } 6239\end{array}$ & & & $*$ & * & \\
\hline SN $1996 \mathrm{~N}$ & $\begin{array}{l}\text { NGC } 1398 \\
16.5 \mathrm{mag}\end{array}$ & $*$ & & * & & \\
\hline SN 1996W & NGC 4027 & & & $*$ & & $*$ \\
\hline SN 1996X & NGC 5061 & & & $*$ & $*$ & \\
\hline
\end{tabular}

to May 9), a total of 25 nights have been allocated to this project. This included a record night when 376 galaxies were observed in just over eight hours. The number of different galaxies included in all of this observing is not known, but is probably around 2000 .

\section{$441 \mathrm{~cm}$ Telescope Performance over the Same Period}

Within this same period of time (Dec. 18, 1994 to the end of April 1996), the $41 \mathrm{~cm}$ backyard telescope played a purely supportive and supplementary role. There is no need to observe any galaxy with this telescope if it has recently been observed with the 40 inch telescope, or was observed as part of the UK Schmidt Supernova Search. With the $41 \mathrm{~cm}$ telescope, all observing is done under fully dark conditions. The working magnitude limit is generally 
about 15.7 mag. The observing conditions near the centre of the township of Coonabarabran are not as good as those on Siding Spring Mountain.

Despite this secondary role, a total of 13,267 observations were made in a period of 231 hours of observing. This made an average rate of galaxy observation of 57.4 galaxies per hour throughout the entire period. Details are listed in Table 2. Of the $41 \mathrm{~cm}$ discoveries, SN 1996X in NGC 5061 was by far the brightest found with either telescope, or by the Perth Observatory group. Of the 40 inch discoveries, SN 1995G in NGC 1643 was also just visible in the $41 \mathrm{~cm}$ telescope, using high magnification, when I already knew where to look for it. It would never have been discovered with this telescope however, as the galaxy was considered to be too distant, and it was not monitored. This supernova has therefore not been included in the results of the $41 \mathrm{~cm}$ search.

\section{Perth Automatic Supernova Search}

This search has been conducted over the last three years by Andrew Williams and Ralph Martin. The $61 \mathrm{~cm}$ telescope is now fully automated, although an observer has to be present to start up and to watch the weather. The magnitude limit during the search was $16 \cdot 5-17 \cdot 0$ (Williams 1997 , present issue p. 208). The figures cover a three-year period starting early in 1993. Figures for either eighteen-month period are not known, although more work was done in the second half than in the first. Each observation normally took four minutes, thus averaging fifteen galaxy observations per hour. A total of 5530 observations were made. The galaxies searched were 300 specially chosen large spiral galaxies in which a higher supernova rate was expected. Originally, there was an expectation that the search would use up to $50 \%$ of available observing time but in practice many other programs were also catered for. These observations yielded five confirmed supernova discoveries, although the Perth observers believed that they found a sixth supernova which could not be confirmed independently before the galaxy disappeared behind the Sun. No other supernovae were seen.

It should be noted that the number of supernovae seen is the true gauge of the effectiveness of a search, in relation to the number of galaxies searched, and not the number of discoveries. The number of these supernovae which are discovered by any group depends upon what competition there is for discoveries at the time, and this may vary from week to week. It has been questioned whether automatic and visual searches can be compared in this way since the automatic searches have a fixed list of targets, while visual observers are likely to be biased in observing a galaxy with a known supernova. In fact, the target list of galaxies for the visual search is also well defined, and I have only included galaxies with known supernovae which were on the target list and so would reasonably be expected to be discovered. In each case, it was clear whether the galaxy would have been observed or not. However, it may well be true that the chances of detecting supernovae are somewhat higher for known supernovae, so the number of supernovae seen should be regarded as an upper limit.

\section{Evaluation}

(a) The Perth search is finding some intrinsically fainter supernovae in the brighter galaxies which were not seen or were otherwise missed in the visual search. The visual search can miss supernovae within one magnitude of the limit $(16 \cdot 5-17 \cdot 5)$ through having to observe in moonlight, a factor which does not degrade CCD images to the same degree. In the visual search, objects at the very faint end have been missed on two occasions that are known, and may have occurred on other occasions. NGC 908 was observed by us a few nights after the Perth group had found SN 1994ae, estimated at mag $16 \cdot 5$. While I knew that a supernova was present I did not know its location within the galaxy, and no new star was seen. Also, NGC 1398 was observed by us several days before the Perth group found SN $1996 \mathrm{~N}$ at mag $16 \cdot 5$. In the first instance, I believe that the supernova was fainter visually than the (red) CCD estimate published. As a result it was not seen by us. In the second instance, the star was seen, but was not recognised as a new object. So, our failure to recognise the new object on this occasion should be put down to human error.

(b) The Perth search's smaller number of observations per supernova seen $(922: 1090: 2211)$ may be due to the particular selection of target galaxies used by them. The visual search covered a much wider range of galaxies, both in type and luminosity. This created a sample of galaxies with a much lower expected supernova rate than the Perth sample.

(c) Allowance must also be made for the fact that $40 \mathrm{inch}$ and $41 \mathrm{~cm}$ figures are only for eighteen months, whereas the Perth figures are for three years. On the average, twice as many supernovae were available to be discovered or seen in a three-year period than in eighteen months.

(d) A surprising result is that the 40 inch search saw a supernova every 41 hours and the $41 \mathrm{~cm}$ search every 38 hours, whereas the Perth computer saw one every 61.5 hours. This difference probably arises from the great difference in the average speed of the Perth search which, in turn, allowed a much wider range of galaxies to be seen by the two visual searches. We were quite surprised that the 40 inch was able to observe galaxies at least $60 \%$ faster than the average speed of the Perth search, despite the fact that the 40 inch does not have the advantage of computerised location of targets. The efficiency 
of the automatic search could be improved by using improved CCDs and faster computers.

(e) Large-telescope visual searches can also be improved by the computerised location of target galaxies. In the case of the 40 inch telescope at Siding Spring the use of the Cassegrain focus port provides a much more efficient use of the telescope than would be possible if a standard Newtonian configuration was being used.

(f) A much longer list of different galaxies needs to be observed regularly with the 40 inch, compared to the smaller telescope, if the number of supernovae to be seen is to be improved and the proper potential of the larger telescope is to be realised. The fainter magnitude limit which is possible with the 40 inch telescope should also be exploited more fully. This, again, emphasises the value of, and need for, a dedicated telescope for this kind of observing.

\section{Conclusion}

(a) The value of visual searching has again been demonstrated, and support for this kind of work should be improved. Especially, visual searching with more modest telescopes is more useful in finding brighter supernovae which, in turn, have a particular value for scientific research at present. However, at this time in the history of astronomy, when supernova studies are playing an important role in so many areas of astrophysical interest (Cappellaro et al. 1996), any efficient method of finding supernovae deserves support. This certainly applies to visual searches using larger telescopes such as the 40 inch, or other telescopes of similar capacity, which can monitor a large number of galaxies over considerable distances.

(b) These figures also indicate that the Perth search was very effective, and probably found all the supernovae that fell within the parameters of its set task. Both types of searching have their own particular merit and role to play. Despite some claims to the contrary, there is no indication that any one method will make the other methods of searching unnecessary. None of the three searches described here would have found all of the supernovae listed. (c) Use of a dedicated telescope is a great advantage, especially in a visual search where access to moonless nights and good weather is more important. It is especially desirable that a dedicated and user-friendly telescope of substantial aperture should be available to prosecute this work more fully and efficiently. The results obtained with the 40 inch telescope were made using only one part in seven of allocatable observing time, including a good percentage of bright moonlight. A great deal more could be achieved if more observing time, including a greater proportion of dark time, were available.

\section{Acknowledgments}

I am very grateful, in the first place, to Dr Russell Cannon, until recently Director of the AngloAustralian Observatory, for his support of this project throughout its history. I am also grateful to the Director of the Mount Stromlo and Siding Spring Observatories, Professor Jeremy Mould, and the Time Allocation Committee for their continuing support, and for the privilege of extensive use of their telescope without financial cost to us. My helpers at the telescope have been numerous. Those who have given more of their time than others are John Shobbrook and Tom Cragg, both of Coonabarabran, and nineteen-year-old Samantha Beaman of Pimpama, Queensland. It is also a pleasure to thank the referees for their many helpful comments.

Note added in proof: Another supernova, SN 1996a1 in NGC 7689, was also discovered with the 40 inch telescope in July 1996, shortly after this paper was prepared.

Cappellaro, E., et al. 1996, A\&A (submitted) Evans, R. 1994, PASA, 11, 7

Muller, R., Newberg, H., Pennypacker, C., Perlmutter, S., Saseen, T., \& Smith, C. 1992, ApJ, 384, L9 van den Bergh, S., \& McClure, R. 1994, ApJ, 425, 205 Williams, A. J. 1997, PASA, 14, 208 